\title{
BMJ Open Neonatal Severe Hyperbilirubinemia Online Registry in Jiangsu Province: protocol for a multicentre, prospective, open, observational cohort study
}

\author{
Qianqian Li (D) , ${ }^{1}$ Xiaoyi Deng, ${ }^{1}$ Junmei Yan, ${ }^{1}$ Xiaofan Sun, ${ }^{2}$ Xiaoyue Dong, ${ }^{2}$ \\ Xiaohui Chen, ${ }^{2}$ Shuping Han, ${ }^{2}$ Jie Huo, ${ }^{3}$ Zhangbin $\mathrm{Yu}^{2}$
}

To cite: Li Q, Deng X, Yan J, et al. Neonatal Severe Hyperbilirubinemia Online Registry in Jiangsu Province: protocol for a multicentre, prospective, open, observational cohort study. BMJ Open 2021;11:e040797. doi:10.1136/ bmjopen-2020-040797

- Prepublication history and additional materials for this paper is available online. To view these files, please visit the journal online (http://dx.doi org/10.1136/bmjopen-2020040797).

Received 22 May 2020

Revised 18 November 2020

Accepted 23 November 2020

Check for updates

(c) Author(s) (or their employer(s)) 2021. Re-use permitted under CC BY-NC. No commercial re-use. See rights and permissions. Published by BMJ.

${ }^{1}$ Neonatology, Xuzhou Maternity and Child Health Care Hospital, Xuzhou, China

${ }^{2}$ Neonatology, Nanjing Maternity and Child Health Care Hospital, Nanjing, China

${ }^{3}$ Neonatology, Yangzhou

Maternity and Child Health Care Hospital, Yangzhou, China

Correspondence to

Dr Zhangbin Yu;

zhangbinyu@njmu.edu.cn

\section{ABSTRACT}

Introduction Severe hyperbilirubinaemia in newborns can be easily complicated by acute bilirubin encephalopathy or even kernicterus, which could lead to neurological sequelae or death. However, there is no systematic study of the management of severe hyperbilirubinaemia in China. The Neonatal Severe Hyperbilirubinemia Online Registry study aims to investigate the management of jaundice before admission, risk factors and outcomes of severe hyperbilirubinaemia in a real-world setting in China. Methods and analysis This is a prospective, multicentre, open, observational cohort study. From May 2020 to April 2023, more than 2000 patients with neonatal severe hyperbilirubinaemia from 13 tertiary hospitals in Jiangsu Province will join the study. Demographic data and treatment information will be collected from their clinical data. Management measures for jaundice before admission will be collected by the WeChat applet (called 'Follow-up of jaundice') after being provided by the patient's guardian using a mobile phone. Follow-up data will include cranial MRI examination results, brainstem auditory-evoked potential or automatic auditory brainstem response, physical examination results and Griffiths Development Scales-Chinese at the corrected ages of 3-6 months and 1 and 2 years. Results and conclusions will be recorded using 'Follow-up of jaundice.' In-hospital outcomes, including severity of hyperbilirubinaemia (severe, extreme, hazardous), acute bilirubin encephalopathy (mild, moderate, severe) and survival status (death or survival), will be collected at discharge. Follow-up outcomes will include loss to follow-up, survival status and kernicterus (yes or no) at 2 years. The research will enhance our comprehensive knowledge of jaundice management before admission, risk factors and outcomes of severe hyperbilirubinaemia in China, which will ultimately help to reduce the incidence of neonatal severe hyperbilirubinaemia.

Ethics and dissemination Our protocol has been approved by the Medical Ethics Committee of Nanjing Maternity and Child Health Care Hospital. We will present our findings at national conferences and peer-reviewed paediatrics journals.

Trial registration number NCT04251286.
Strengths and limitations of this study

- The Neonatal Severe Hyperbilirubinemia Online Registry is the first multicentre, prospective, open, observational cohort study with a maximum followup of 2 years in China.

- This is the first registry study aiming to systematically investigate the outcomes of patients with severe hyperbilirubinaemia in a real-world setting in China.

- For the first time, this study will investigate the implementation of jaundice management measures before admission for patients with severe hyperbilirubinaemia in China.

- It is possible that some participants will be lost to follow-up because of the observational nature of this study.

\section{INTRODUCTION}

Approximately $70 \%$ of healthy term and late-preterm newborns have jaundice during the first week of life. ${ }^{1}$ Hyperbilirubinaemia without standardised monitoring and treatment may rapidly develop into severe hyperbilirubinaemia, and bilirubin-induced neurotoxicity usually results in acute bilirubin encephalopathy (ABE) or kernicterus, leading to neurological sequelae or death. ${ }^{2}$ Severe hyperbilirubinaemia and its complications have been reported in most areas of the world, such as Asia, Africa, the Middle East, ${ }^{3-6}$ Australia $^{7}$ and the USA. $^{8}$ Mortality due to severe hyperbilirubinaemia has attracted the attention of researchers. The Global Burden of Disease 2016 study reported that neonatal jaundice ranks seventh and ninth globally for all causes of death for early (0-6 days) and late (7-27 days) newborns, respectively. ${ }^{9} 10$

Many countries have realised the damage of severe hyperbilirubinaemia and its complications, but only a few countries have established a national-level registry to determine 
the causes and definite prognosis. In 2009, the USA released a report of the kernicterus pilot registry, which indicated that the main cause of kernicterus is the failure to detect high-risk infants earlier. No case of kernicterus in healthy infants has been reported when total serum bilirubin (TSB) levels are $<20 \mathrm{mg} / \mathrm{dL}(342 \mu \mathrm{mol} / \mathrm{L}) .{ }^{211} \mathrm{In}$ 2012, because 11 cases of kernicterus in term infants were reported over the course of 10 years, the Italian Society of Neonatology decided to develop the national registry of kernicterus and hyperbilirubinaemia for newborns. ${ }^{12}$ Furthermore, after the establishment of the online registration of neonatal jaundice in Turkey, a prospective study of healthy infants indicated that $13(0.23 \%)$ had $\mathrm{ABE}$, including two patients who died during hospitalisation and others with posticteric sequela at 3 months after discharge. $^{13}$

In China, there has been no comprehensive study of the causes and outcomes of severe hyperbilirubinaemia. A multicentre retrospective study published in 2012 showed that only 223 cases of 348 children with bilirubin encephalopathy or kernicterus were followed up; of these children, 36 died. ${ }^{14}$ A large number of missing cases without follow-up rendered the prognosis analysis incomplete. Additionally, China issued The Experts Consensus on the Management of Neonatal Hyperbilirubinemia, ${ }^{15}$ which formulated the management of jaundice after discharge from delivery institutions. At the same time, our team established and validated a risk stratification model for predicting hyperbilirubinaemia after discharge of healthy term and late-preterm infants. ${ }^{16}$ These measures were hoped to reduce the incidence of severe hyperbilirubinaemia, $\mathrm{ABE}$ and kernicterus through standardised management. However, little research has systematically investigated the completion of management measures for jaundice before admission and the risk factors for infants with severe hyperbilirubinaemia in China, and these are very important for reducing the incidence of severe hyperbilirubinaemia.

Knowledge of the management measures for jaundice before admission, risk factors and outcomes of severe hyperbilirubinaemia are not comprehensive in the realworld setting in China. Accordingly, we designed a multicentre, prospective, open, observational cohort study to investigate these factors in a real-world setting in Jiangsu Province to help reduce the incidence of severe hyperbilirubinaemia, ABE and kernicterus. Furthermore, we established the Neonatal Severe Hyperbilirubinemia Online Registry and 'Follow-up of jaundice' (WeChat applet) to achieve these goals.

\section{OBJECTIVE}

The primary objective of the registry is to investigate the comprehensive prognosis of infants with severe hyperbilirubinaemia at the corrected age of 2 years in a real-world setting in Jiangsu Province.

Secondary objectives of this project will be to: investigate the implementation of jaundice management

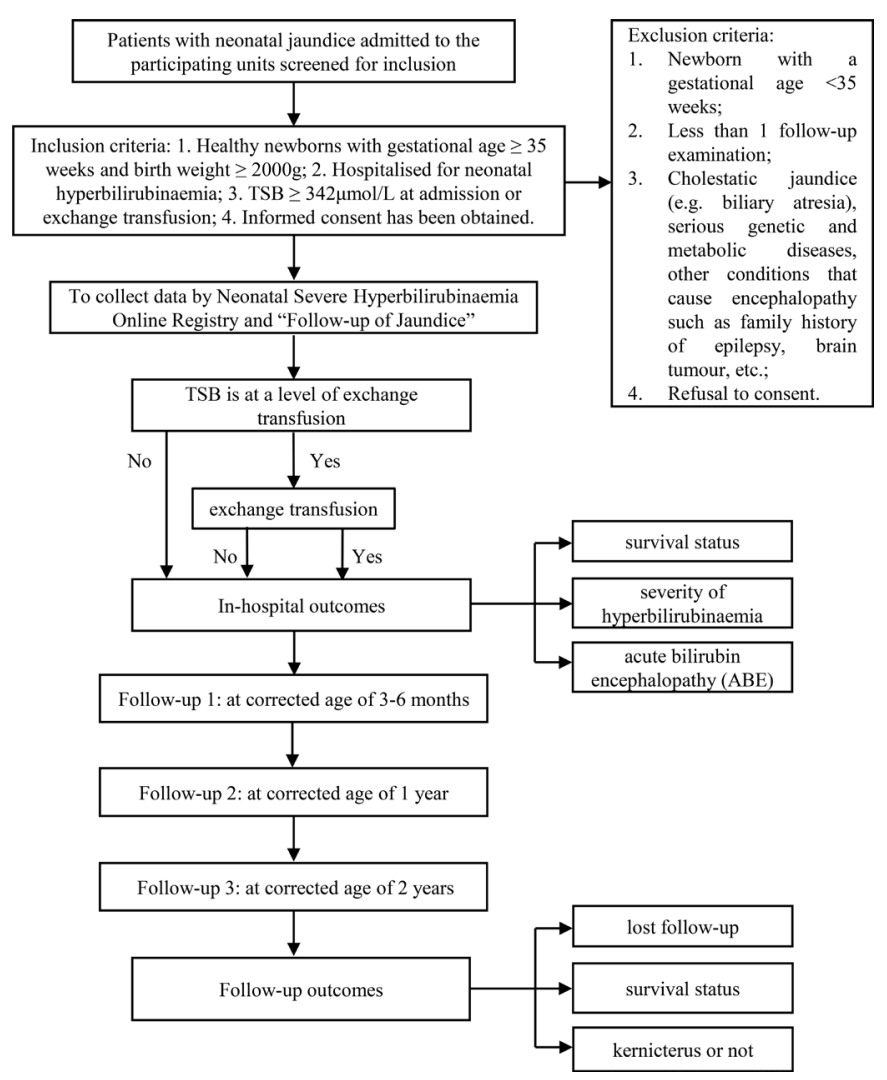

Figure 1 Flow chart of the study process to determine the causes, management and outcomes of severe hyperbilirubinaemia. TSB, total serum bilirubin.

measures before admission for patients with severe hyperbilirubinaemia; analyse risk factors for extreme hyperbilirubinaemia (TSB level $\geq 427 \mu \mathrm{mol} / \mathrm{L}$ ) because an infant with this degree of jaundice is thought to be at high risk for kernicterus ${ }^{17}$; and assess the clinical outcome at 2 years of corrected age of cases treated with and without exchange transfusion when TSB is at the threshold level.

\section{METHODS \\ Study design}

This study is a multicentre, prospective, open, observational cohort study that will be conducted from May 2020 to April 2023. More than 2000 patients with severe hyperbilirubinaemia will be recruited from 13 participating hospitals. Follow-up for a maximum of 2 years will be performed after discharge of all enrolled infants. The protocol has been approved by the local and regional ethics committee at the coordinating centre. All participating hospitals will be asked to treat patients according to Management of Hyperbilirubinemia in the Newborn Infant 35 or More Weeks of Gestation by the American Academy of Pediatrics. ${ }^{18}$ Figure 1 is a summary of the study process.

Nanjing Maternity and Child Health Care Hospital designed and launched the study. It is the coordinating centre and the affiliation of the promoter $(\mathrm{ZY})$ and coordinator and primary investigator (QL). To improve the universality of the results, an additional 12 centres 
across Jiangsu Province have been invited to participate in the study, and each centre has more than 2000 births each year and extensive experience in the treatment of neonatal severe hyperbilirubinaemia and exchange transfusion. A primary data collection recorder (newborn paediatrician) and a data checker (newborn paediatrician) were appointed at each participating centre. We warmly welcome centres that meet the relevant standards to join the study during the recruitment stage because of the open nature of the study. We drafted this protocol adhering to the Standardized Protocol Items: Recommendations for Observational Studies statement (online supplemental file 1).

\section{Patient and public involvement}

Patients and the public did not participate in the study design. Patients will participate in the recruitment stage of the study. Participation is entirely voluntary. The study results will be disseminated to guardians and the public through public health education and neonatal jaundice academic conferences.

\section{Participants}

Thirteen hospitals have formed a multicentre research cooperation group for severe hyperbilirubinaemia in Jiangsu Province. All infants with severe hyperbilirubinaemia admitted to these hospitals from 1 May 2020 to 30 April 2023 will be selected for analysis. Clinical practice will not be affected by patients who refuse to participate in or withdraw from the study.

\section{Criteria}

Inclusion criteria are as follows:

1. Healthy newborn with a gestational age $\geq 35$ weeks and birth weight $\geq 2000 \mathrm{~g}$.

2. Hospitalised for neonatal hyperbilirubinaemia.

3. TSB $\geq 342 \mu \mathrm{mol} / \mathrm{L}$ at admission or exchange transfusion.

4. Informed consent has been obtained.

Exclusion criteria are as follows:

1. Newborn with a gestational age $<35$ weeks.

2. Less than one follow-up examination.

3. Cholestatic jaundice (eg, biliary atresia), serious genetic and metabolic diseases, other conditions that cause encephalopathy such as family history of epilepsy, brain tumour, and so on.

4. Refusal to consent.

\section{Standardisation of definitions of essential variables}

Definitions of commonly reported essential variables were standardised. Severe hyperbilirubinaemia was defined when the peak value of TSB was $\geq 342 \mu \mathrm{mol} / \mathrm{L}$ or exchange transfusion. ${ }^{11}$ The severity of hyperbilirubinaemia was classified as follows: severe, TSB levels $\geq 342$ $\mu \mathrm{mol} / \mathrm{L}(20 \mathrm{mg} / \mathrm{dL})$; extreme, TSB levels $\geq 427 \mu \mathrm{mol} / \mathrm{L}$ $(25 \mathrm{mg} / \mathrm{dL})$; and hazardous, TSB levels $\geq 513 \mu \mathrm{mol} / \mathrm{L}$ $(30 \mathrm{mg} / \mathrm{dL}) .{ }^{19} \mathrm{ABE}$ was used to describe the acute clinical symptoms of bilirubin neurotoxicity during the first few weeks after birth, ${ }^{18}$ including mild (poor feeding, lethargy and tone abnormalities), moderate (highpitched cry, irritability and increasing hypertonia) and severe (deep stupor, apnoea, inability to feed, fever, retrocollis, opisthotonos and obtundation). ${ }^{10}$ The bilirubininduced neurological dysfunction (BIND) score is used to describe the progression of ABE: scores 1-3, mild ABE; scores 4-6, moderate ABE; and scores 7-9, severe ABE. ${ }^{11} 20$ Kernicterus is described as chronic neurological sequelae induced by bilirubin toxicity ${ }^{18}$; this definition, which we made minor changes to, was taken from the study by Sgro $e t a l .^{21}$ Infants are diagnosed with kernicterus if they satisfy either of two definitions. The first definition is a history of severe hyperbilirubinaemia as a newborn (TSB level at admission $\geq 324 \mu \mathrm{mol} / \mathrm{L}(20 \mathrm{mg} / \mathrm{dL})$ or exchange transfusion) and at least two of the following neurological sequelae: extrapyramidal disorders (eg, athetosis, dystonia); other movement disorders (hypotonia or spasticity); gaze abnormalities; sensorineural hearing loss; intellectual deficits; and enamel dysplasia. The second definition included a history of severe hyperbilirubinaemia as a newborn (TSB level at admission $\geq 324$ $\mathrm{mol} / \mathrm{L}(20 \mathrm{mg} / \mathrm{dL}))$ and abnormal MRI results defined as bilateral lesions of the basal ganglia/midbrain (globus pallidus and subthalamic nucleus). Corrected age: for each preterm infant, the corrected age is calculated by subtracting the number of weeks of preterm birth from the chronological age after birth (40 weeks minus actual gestational age) ${ }^{22}$ Sepsis: it is defined as confirmed sepsis (culture positive) or clinical sepsis (culture negative, but other infection indicators are elevated, such as white cell count, immature to total neutrophil ratio, C-reaction protein, procalcitonin, and so on), and treatment with antibiotics throughout the course ${ }^{11}$ Haemolytic disease: haematocrit $\leq 40 \%$ (anaemia) within 2 weeks, increased reticulocyte count and corroborating signs such as positive direct antiglobulin test (DAT). ${ }^{11}$ Dehydration: birthweight loss $\geq 15 \%$ and/or serum sodium $>150 \mathrm{mmol} / \mathrm{L}$ on admission. $^{11}$

\section{Informed consent}

Written informed consent will be obtained from the patients' guardians. This study complies with the Declaration of Helsinki.

\section{Data collection and management}

This study will recruit patients in a prospective manner, and the recruitment phase is expected to be closed in 2023. Each participating centre and its patients have unique identification numbers. Data collection for this study will be performed online in two parts. Demographic, diagnosis, treatment and discharge information will be collected based on the Neonatal Severe Hyperbilirubinemia Online Registry using the online database of Ningbeixin newborn homogeneity collaborative research platform (https://www.ningbx.com/), which has been established by our collaboration group. Furthermore, data collection is the standard of care for these patients. All data in the online database will be entered by primary 
Table 1 Data collection by 'Follow-up of jaundice'

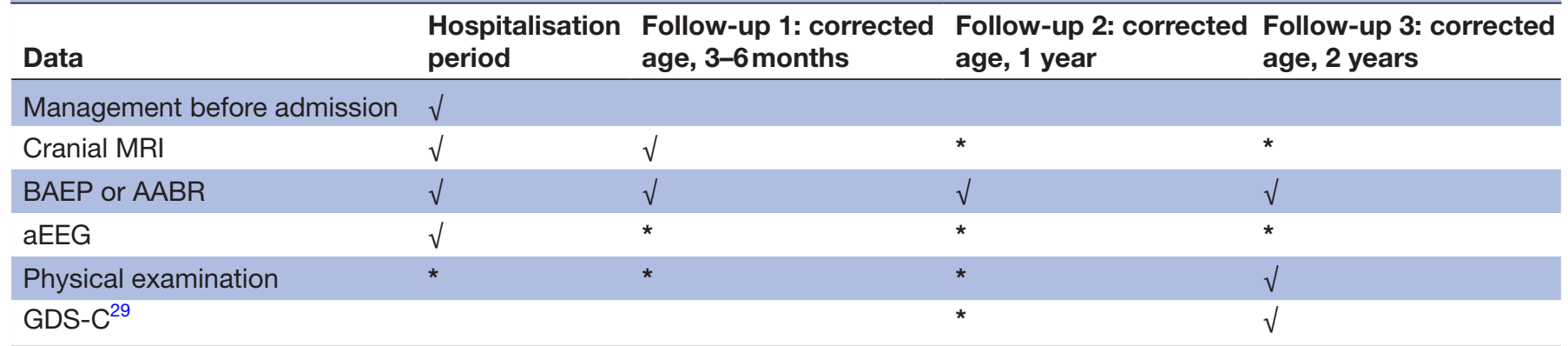

$\sqrt{ }$ Required. *If applicable.

AABR, automatic auditory brainstem response; aEEG, amplitude-integrated electroencephalogram; BAEP, brainstem auditory-evoked potential; GDS-C, Griffiths Development Scales-Chinese.

data collection recorders who have been trained to perform database entry and have obtained entry qualifications; the data checker will regularly perform quality control and provide feedback regarding the data. The online database can modify and update the information in a timely manner, and it can export the data for quality control and statistical analysis in real time. Furthermore, the cooperation group developed the "Follow-up of jaundice' (WeChat applet) for patients with severe hyperbilirubinaemia; this can more accurately collect the management information for jaundice before admission and the examination results during follow-up (table 1). This applet is divided into the physician port and guardian port; the guardian can provide information using the guardian port of a mobile phone under the guidance of a physician. If the guardian fails to provide results using the applet when the child is 2 years old, then we will perform a telephone interview. All of these measures will ensure the authenticity of the data and increase the participation of the guardians. Furthermore, the data are inter-related and shared to reduce input errors. Online supplemental file 2 describes the specific process. We recommend that hospitals with equipment and conditions perform brainstem auditory-evoked potential (BAEP) and cranial MRI examinations during hospitalisation for infants with severe hyperbilirubinaemia who meet the inclusion criteria, and try to improve follow-up examinations according to the plan after discharge. Medical insurance will cover part of the cost of the examination during hospitalisation for insurant.

The coordination centre will have access to the information and data, which will be analysed and published in an anonymised form. Additionally, the coordination centre will ensure the management and operation of the online registry and WeChat applet, as well as data security. The data monitoring committee consists of the principal investigator of the coordination centre and chief investigators of 12 participating hospitals, and it will be responsible for monitoring the conduct of the study to ensure compliance with the study protocol. The data monitoring committee will submit a modification plan for the study design to the ethics committee, if necessary, and implement the modification after approval. Quality control and interim analyses meetings will occur regularly during the study to assess the progress of the study and whether there is any protocol deviation. The committee is also responsible for other aspects, including recruitment of participating hospitals, providing study materials, organising training meetings, checking and analysing data, and publishing study reports. The ethics committee, which is independent of the sponsor and investigators, will audit the conduct of the study annually.

\section{Data elements}

To achieve our goals, we have carefully designed an online registry for neonatal severe hyperbilirubinaemia and 'Follow-up of jaundice' (WeChat applet) to collect necessary information (online supplemental file 3 ), which will include the following:

1. Admission process: birth hospital, birth time, admission mode (admission to rooming-in care, outpatient).

2. Perinatal information:

- Obstetric information: mother's name and mobile phone number for follow-up use, fetal number, sex, age, birth weight, head circumference, length, Apgar score (1 min, 5 min, $10 \mathrm{~min}$ ).

- Parents' information: parents' age, ABO blood type, Rh blood type, education level and nationality.

- Perinatal situation: gravidity and parity history, delivery mode, maternal hypertension, diabetes, intrahepatic cholestasis during pregnancy, hypothyroidism during pregnancy, number of children, phototherapy or exchange transfusion of previous children.

3. Diagnosis of and treatment information for severe hyperbilirubinaemia:

- Basic information: name, date of admission, age at admission, weight at admission and temperature at admission.

- Pathogeny of hyperbilirubinaemia: infection (sepsis, other inflammation such as pneumonia, TORCH infection(Toxopasma, Rubellavirus, Cytomegalovirus, Herpessimplxvirus), and so on), haemorrhagic diseases (head haematoma, intracranial haemorrhage, and so on), haemolytic diseases ( $\mathrm{ABO}$ haemolysis, 
Rh haemolysis, glucose-6-phosphate dehydrogenase deficiency, and so on), others (ecchymosis of skin, breast milk jaundice, dehydration, metabolic acidosis, hypothyroidism, total parenteral nutritionrelated cholestasis, genetic factors).

- Examination indexes: DAT, total transcutaneous bilirubin at admission, the highest TSB value and time, the start time and hours of phototherapy (bilirubin is measured before the start of phototherapy; the TSB is measured at admission, the transcutaneous bilirubin value is measured at least twice a day in the later stage and the TSB is measured when necessary), direct bilirubin, alanine aminotransferase, aspartate transaminase, albumin, haemoglobin, haematocrit, the total times and hours of phototherapy, exchange transfusion.

- Treatment data, including types of drugs, such as antibiotics, intravenous immunoglobulin, albumin, phenobarbital, and others.

- The BIND score: the scoring time and corresponding scores.

- Management information regarding jaundice before admission: discharge time at delivery institution, jaundice education of guardian during pregnancy and before discharge, parents' knowledge of hyperbilirubinaemia hazards, outpatient follow-up reminder from physicians before discharge according to jaundice risk, follow-up methods for medical reminders after discharge, whether the newborn is followed up according to the requirements, admission mode during follow-up (follow-up according to the plan, found by oneself, found by physicians onsite, phone notification), the number of follow-up visits between discharge from delivery institutions and admission.

- Auxiliary examination information: BAEP or automatic auditory brainstem response (AABR), cranial MRI, amplitude-integrated electroencephalogram, physical examination and Griffiths Development Scales-Chinese (GDS-C).

- Discharge conditions: severity of hyperbilirubinaemia, bilirubin neurotoxicity, whether there are symptoms of $\mathrm{ABE}$ at admission, the time of $\mathrm{ABE}$ occurrence, time of discharge, weight at discharge, prognosis at discharge (improved or cured, requiring discharge, death due to hyperbilirubinaemia or not), cost.

4. Follow-up examination: cranial MRI, BAEP or AABR and physical examination results at the corrected age of 3-6 months, BAEP or AABR and physical examination results at the corrected ages of 1 and 2 years, GDS-C at the corrected age of 2 years (table 1 ).

5. Follow-up conclusion: this is an important part of the diagnosis of kernicterus; therefore, it should be completed after 18-24 months of follow-up. It includes loss to follow-up, evaluation date, kernicterus category, kernicterus severity, cerebral palsy, epilepsy, cognitive impairment, hearing impairment, eye movement disorder, extrapyramidal motor disorder, enamel dysplasia, motor retardation, neurodevelopmental delay and other (eg, death). This part of diagnosis needs to be confirmed jointly by the paediatric neurologist and paediatric rehabilitation physician.

\section{Clinical outcomes}

In-hospital outcomes include severity of hyperbilirubinaemia (severe, extreme, hazardous), ABE (mild, moderate, severe) and survival status (death or survival) at discharge. Follow-up outcomes will include loss to follow-up, survival status and kernicterus (yes or no) at 2 years.

\section{Sample size estimation}

The primary objective is to comprehensively describe the prognosis of infants with severe hyperbilirubinaemia, which usually does not require estimation of the sample size because of the observational nature of the study. Therefore, the sample size for the logistic analysis of risk factors for extreme hyperbilirubinaemia will be calculated. We expect to test $5-10$ variables as potential risk factors; therefore, we will need 100 infants with extreme hyperbilirubinaemia according to the criteria of at least 10 events per variable. ${ }^{23}{ }^{24}$ Based on our previous study (Multicenter Research Collaboration Group on Severe Hyperbilirubinemia in Jiangsu Province, Survey of severe hyperbilirubinaemia in Jiangsu Province), the rate of extreme hyperbilirubinaemia in severe hyperbilirubinaemia is $16.2 \%$; therefore, a minimum sample size of 741 patients with severe hyperbilirubinaemia is required, assuming a loss to follow-up rate of $20 \%$. We expect to recruit more than 2000 patients for the study.

\section{Statistical analysis}

The patient's gestational age, TSB values, admission age, phototherapy duration, and so on will be presented as mean (SD) or median (range), while prognosis such as in-hospital outcomes and follow-up outcomes and the implementation of jaundice management measures before admission will be presented as frequencies or percentages. Different results of demographics, prognosis and implementation of jaundice management measures among patient groups will be compared with independent Student's t-test or Mann-Whitney U test results for continuous variables, and $\chi^{2}$ test or Fisher's exact test results for categorical variables. A univariate regression analysis will be used to evaluate the association between implementation of jaundice management measures or exchange transfusion and prognosis. An analysis of risk factors for extreme hyperbilirubinaemia will be used for multivariate logistic regression. In order to reduce systematic differences in the results, we will conduct an overall analysis of the data and separate analysis of the participating centres for the analysis of the implementation of jaundice management measures. $P$ values of 0.05 will be considered statistically significant. 


\section{DISCUSSION}

Some countries have established jaundice registry to find the root cause, ${ }^{11} 13$ or formulated guidelines for the management of jaundice for the corresponding population, ${ }^{25} 26$ or formulated further research plans. ${ }^{27}$ The special disease registry has the advantage of quickly garnering a large amount of data and using the data to develop quality improvement plans, ${ }^{28}$ so we designed the Neonatal Severe Hyperbilirubinemia Online Registry in Jiangsu Province

This protocol describes the Neonatal Severe Hyperbilirubinemia Online Registry in Jiangsu Province, which is the first multicentre, prospective, open, observational cohort study to investigate the management measures for jaundice before admission, risk factors and outcomes of severe hyperbilirubinaemia in a real-world setting in Jiangsu Province in China. Recording the systematic prognosis more comprehensively will help us gain a comprehensive understanding of the outcomes of severe hyperbilirubinaemia. This study will also investigate management measures for jaundice before the admission of infants with severe hyperbilirubinaemia for the first time in China, and it will inform which management measures have problems when implemented in a realworld setting, thereby providing a basis for correcting deficiencies. By exploring the risk factors for extreme hyperbilirubinaemia, the study will suggest intensive monitoring of at-risk infants to prevent the occurrence of extreme hyperbilirubinaemia. Furthermore, this study will also provide long-term prognosis information for patients with or without exchange transfusion when TSB is at the threshold level to optimise individualised treatment of such patients.

The limitations of observational studies are inevitable, such as a high number of participants lost to follow-up. To overcome these limitations, we suggest that guardians use their mobile phones to participate in follow-up using the WeChat applet under the guidance of the physician. Furthermore, if the guardian fails to provide follow-up results when the infant is 2 years old, then we will perform a telephone interview.

In conclusion, this prospective study will improve the understanding of inadequate management before admission and provide a comprehensive prognosis for infants with severe hyperbilirubinaemia. Ultimately, it will help prevent the occurrence of severe hyperbilirubinaemia and optimise personalised intervention and therapeutic schedules.

\section{ETHICS AND DISSEMINATION}

Our protocol has been approved by the Medical Ethics Committee of Nanjing Maternity and Child Health Care Hospital. Each participating centre needs to be approved by the local ethics committee, and its ethics approval must be provided before each centre starts recruitment. All patient guardians will be provided with a full understanding of the study. They will obtain written information and sign a consent form prior to recruitment. Hospitals participating in the study will share the findings and results of the study, which will be presented at national conferences and peer-reviewed paediatrics journals.

Acknowledgements There are 13 tertiary hospitals in Jiangsu Province in Eastern China participating in the study, and we would like to thank data collection recorders and data checkers of them, including Nanjing Maternity and Child Health Care Hospital and Women's Hospital of Nanjing Medical University (Jun Zhang and ZY), Xuzhou Maternity and Child Health Care Hospital (Zengqin Wang and $X D e)$, Jiangsu Women and Children Health Hospital (Yujie Yin and Xiaoging Chen), Changzhou Maternity and Child Health Care Hospital (Jinxiu Wang and Huaiyan Wang), Lianyungang Maternity and Child Health Care Hospital (Lin Bai and Yan Gao), Affiliated Hospital of Jiangsu University (Yu Qiao and Hongyan Lu), Suqian First Hospital (Bin Tang and Jia Zhang), Jiangyin People's Hospital (Weimin Wu and Jun Wan), Nantong Maternity and Child Health Care Hospital (Jinjun Zhou and Shuangshuang Li), Affiliated Hospital of Yangzhou University (Fudong Wang and Mingfu Wu), Yangzhou Maternity and Child Health Care Hospital (JH and Xinping Wu), The Affiliated Hospital of Xuzhou Medical University (Yan Xu and Jun Wang), Taizhou People's Hospital (Li Yang and Mei Xue).

Contributors ZY and SH planned the study. ZY and XDe designed the study. ZY and QL provided ethical support and registered the study. QL, JY and XDe wrote the study protocol and revised the manuscript. SH, XDo and XC performed a critical revision of the manuscript. XS and $\mathrm{JH}$ provided the statistical analysis. All the authors read and approved the final version.

Funding This work was supported by Top Talent Research Project of 'Six One Project' of Jiangsu Province (grant number LGY2017004), Key Talent of Maternal and Child Health of Jiangsu Province (grant number FRC201733) and Key Research and Development Plan (Social Development) of Jiangsu Province (grant number BE2019620).

Disclaimer Study sponsor and funders play no role in design and management of the study, collection, analysis and interpretation of data, writing of the report or the decision on submitting the report for publication.

Competing interests None declared.

Patient consent for publication Not required.

Ethics approval Central ethics approval is obtained from the Medical Ethics Committee of Nanjing Maternity and Child Health Care Hospital (Reference No (2020) KY-014).

Provenance and peer review Not commissioned; externally peer reviewed.

Supplemental material This content has been supplied by the author(s). It has not been vetted by BMJ Publishing Group Limited (BMJ) and may not have been peer-reviewed. Any opinions or recommendations discussed are solely those of the author(s) and are not endorsed by BMJ. BMJ disclaims all liability and responsibility arising from any reliance placed on the content. Where the content includes any translated material, BMJ does not warrant the accuracy and reliability of the translations (including but not limited to local regulations, clinical guidelines, terminology, drug names and drug dosages), and is not responsible for any error and/or omissions arising from translation and adaptation or otherwise.

Open access This is an open access article distributed in accordance with the Creative Commons Attribution Non Commercial (CC BY-NC 4.0) license, which permits others to distribute, remix, adapt, build upon this work non-commercially, and license their derivative works on different terms, provided the original work is properly cited, appropriate credit is given, any changes made indicated, and the use is non-commercial. See: http://creativecommons.org/licenses/by-nc/4.0/.

ORCID iD

Qianqian Li http://orcid.org/0000-0002-1676-131X

\section{REFERENCES}

1 Draque CM, Sañudo A, de Araujo Peres C, et al. Transcutaneous bilirubin in exclusively breastfed healthy term newborns up to 12 days of life. Pediatrics 2011;128:e565-71.

2 Bhutani VK, Johnson L. Synopsis report from the pilot USA kernicterus registry. J Perinatol 2009;29:S4-7.

3 Bhutani VK, Zipursky A, Blencowe H, et al. Neonatal hyperbilirubinemia and rhesus disease of the newborn: incidence 
and impairment estimates for 2010 at regional and global levels. Pediatr Res 2013;74:86-100.

4 Diala UM, Wennberg RP, Abdulkadir I, et al. Patterns of acute bilirubin encephalopathy in Nigeria: a multicenter pre-intervention study. J Perinatol 2018;38:873-80.

5 Greco C, Arnolda G, Boo N-Y, et al. Neonatal jaundice in lowand middle-income countries: lessons and future directions from the 2015 Don Ostrow Trieste yellow retreat. Neonatology 2016;110:172-80.

6 Iskander I, Gamaleldin R, El Houchi S, et al. Serum bilirubin and bilirubin/albumin ratio as predictors of bilirubin encephalopathy. Pediatrics 2014;134:e1330-9.

7 McGillivray A, Evans N. Severe neonatal jaundice: is it a rare event in Australia? J Paediatr Child Health 2012;48:801-7.

8 Christensen RD, Agarwal AM, George TI, et al. Acute neonatal bilirubin encephalopathy in the State of Utah 2009-2018. Blood Cells Mol Dis 2018;72:10-13.

9 Olusanya BO, Teeple S, Kassebaum NJ. The contribution of neonatal jaundice to global child mortality: findings from the GBD 2016 study. Pediatrics 2018:141 doi:10.1542/peds.2017-1471

10 Olusanya BO, Kaplan M, Hansen TWR. Neonatal hyperbilirubinaemia: a global perspective. Lancet Child Adolesc Health 2018;2:610-20.

11 Johnson L, Bhutani VK, Karp K, et al. Clinical report from the pilot USA kernicterus registry (1992 to 2004). J Perinatol 2009;29 Suppl 1:S25-45.

12 Pratesi S, Dani C, Raimondi F, et al. The Italian registry of kernicterus and hyperbilirubinaemia. J Matern Fetal Neonatal Med 2012;25:110-2.

13 Erdeve O, Okulu E, Olukman O, et al. The Turkish neonatal jaundice online registry: a national root cause analysis. PLoS One 2018;13:e0193108.

14 Subspecialty Group of Neonatology,, Society of Pediatrics,, Chinese Medical Association. Chinese multicenter study coordination group for neonatal bilirubin encephalopathy. clinical characteristics of bilirubin encephalopathy in Chinese newborn infants-a national multicenter survey. Zhonghua Er Ke Za Zhi 2012;50:331-5.

15 . The experts consensus on the management of neonatal hyperbilirubinemia]. Zhonghua Er Ke Za Zhi 2014;52:745-8.

16 Han S, Yu Z, Liu L, et al. A model for predicting significant hyperbilirubinemia in neonates from China. Pediatrics 2015;136:e896-905.
17 Bhutani VK, Johnson LH. Urgent clinical need for accurate and precise bilirubin measurements in the United States to prevent kernicterus. Clin Chem 2004;50:477-80.

18 American Academy of Pediatrics Subcommittee on Hyperbilirubinemia. Management of hyperbilirubinemia in the newborn infant 35 or more weeks of gestation. Pediatrics 2004:114:297-316. doi:10.1542/peds.114.1.297

19 Bhutani VK, Johnson LH, Jeffrey Maisels M, et al. Kernicterus: epidemiological strategies for its prevention through systems-based approaches. J Perinatol 2004;24:650-62.

20 Johnson L, Brown AK, Bhutani VK. BIND - a clinical score for bilirubin induced neurological dysfunction in newborns. Pediatrics1999;104:746-7.

21 Sgro M, Campbell DM, Kandasamy S, et al. Incidence of chronic bilirubin encephalopathy in Canada, 2007-2008. Pediatrics 2012;130:e886-90

22 Zhao Z, Ding M, Hu Z, et al. Trajectories of length, weight, and bone mineral density among preterm infants during the first 12 months of corrected age in China. BMC Pediatr 2015;15:91.

23 Harrell FE, Lee KL, Mark DB. Multivariable prognostic models: issues in developing models, evaluating assumptions and adequacy, and measuring and reducing errors. Stat Med 1996:15:361-87.

24 Peduzzi P, Concato J, Kemper E, et al. A simulation study of the number of events per variable in logistic regression analysis. $J$ Clin Epidemiol 1996:49:1373-9.

25 Romagnoli C, Barone G, Pratesi S, et al. Italian guidelines for management and treatment of hyperbilirubinaemia of newborn infants $\geq 35$ weeks' gestational age. Ital J Pediatr 2014;40:11.

26 Çoban A, Türkmen MK, Gürsoy T. Turkish neonatal Society guideline to the approach, follow-up, and treatment of neonatal jaundice. Turk Pediatri Ars 2018;53:172-9.

27 Bahr TM, Christensen RD, Agarwal AM, et al. The neonatal acute bilirubin encephalopathy registry (NABER): background, aims, and protocol. Neonatology 2019;115:242-6.

28 Schleelein LE, Harris KA, Elliott EM. Developing multicenter registries to advance quality science. Anesthesiol Clin 2018;36:75-86.

29 Tso WWY, Wong VCN, Xia X, et al. The Griffiths Development ScalesChinese (GDS-C): a cross-cultural comparison of developmental trajectories between Chinese and British children. Child Care Health Dev 2018;44:378-83. 